\title{
Simultaneous Arterial and Venous Brain Infarctions in a Patient with COVID-19: Case Report
}

\author{
Sonia Bermúdez ${ }^{1}$ Paula Andrea Forero ${ }^{1} \cdot$ Vanessa Salej $^{1} \cdot$ Silvia González $^{1} \cdot$ Jaime Toro $^{1}$
}

Accepted: 9 September 2021 / Published online: 21 September 2021

(c) The Author(s), under exclusive licence to Springer Nature Switzerland AG 2021

\begin{abstract}
Stroke is one of the manifestations of COVID-19-associated coagulopathy. Arterial infarcts are the most common presentation; however involvement of both arterial and venous irrigation is possible but rare. We report, what is, to our knowledge, the second case of concomitant arterial and venous brain thrombosis evidenced in magnetic resonance. A 62-year-old man presented with acute weakness of the left hand and lack of coordination in the left arm. Nine days earlier, he was positive for SARS-CoV-2 RT-PCR. The brain images revealed two subacute infarcts, one corresponding to the territory of the right middle cerebral artery, and the other in the right frontal cortical vein. The existence of both venous and arterial brain infarcts due to COVID-19 infection, has been previously reported once. Most of the cases of stroke are due to only arterial thrombosis; therefore, this could be the starting point to start collecting data about simultaneous compromise in order to assess and compare outcomes, severity of the disease, among other variables.
\end{abstract}

Keywords Stroke $\cdot$ Vascular disease $\cdot$ COVID-19 - Arterial and venous infarct

\section{Introduction}

Since the emergence of COVID-19, multiple clinical outcomes have been described, among them the development of a hypercoagulability state, also called COVID-19-associated coagulopathy (CAC) [1]. This occurs due to a dysfunction in the three components of Virchow's triad, which induces the formation of clots in either arterial or venous circulation, mostly manifesting as a ischemic stroke, pulmonary thromboembolism, or deep vein thrombosis [1]. Involvement of

This article is part of the Topical Collection on Covid-19.

Paula Andrea Forero

paulaforero1129@gmail.com

Sonia Bermúdez

sonia.bermudez@radiologiafsfb.org

Vanessa Salej

vanessa.salej@gmail.com

Silvia González

silvia9007@gmail.com

Jaime Toro

jaimetorog1@gmail.com

1 Fundación Santa Fe de Bogotá, 116 street \# 9-02, 110111 Bogota, Columbia both arterial and venous irrigations at any part of the body is possible but rare; in this setting we report, what is, to our knowledge, the second case of concomitant arterial and venous brain thrombosis, evidenced in a magnetic resonance angiography (MRA) of the brain, in a patient with COVID19 diagnosis [2].

\section{Report of a Case}

A 62-year-old man presented to the emergency department of an institution in Aruba with acute weakness of the left hand and lack of coordination of the left arm. He had tested positive for SARS-CoV-2 on a real-time reverse transcription polymerase chain reaction assay (RT-PCR) of nasopharyngeal swab 9 days earlier. He had not been vaccinated against COVID-19. The patient's medical history was relevant for a former smoker for 43 years and psoriasis treated with methotrexate. On the examination, the patient showed drooping of the commissure of the left lip, and predominantly distal weakness and dysdiadochokinesia of the left arm. An unenhanced head computed tomography (CT) and a head CT angiogram were performed. The CT results showed hypodense areas in the right parietal and occipital lobes reported as "in an arterial distribution," and 
increased signal at the right transverse and sigmoid dural sinuses, as well as the right internal jugular vein, findings which were confirmed by a filling defect in the CT angiogram. A diagnosis of dural sinus thrombosis was made, and the patient was treated initially with low molecular weight heparin. Two days later, anticoagulation was switched to apixaban at a dose of $5 \mathrm{mg}(\mathrm{mg})$ twice daily. After discharge, 1 month later, the patient was referred to our consult in Bogotá, Colombia for follow-up, where additional studies were indicated. Among the studies performed, the laboratory tests excluded disseminated intravascular coagulation, and other causes of hypercoagulability. Magnetic resonance (MR) imaging of the brain and MR angiography were also requested, which showed two subacute infarcts; one in the arterial territory of the right middle cerebral artery, with triangular shape and with cortical enhancement, and the other in the subcortical venous area, associated with thrombosis of the frontal cortical vein of the same side, and hemorrhage (Fig. 1). Also, partial recanalization of the transverse sinus and right jugular vein thrombosis were seen (Fig. 2). The patient recovered from his initial deficit and currently has no neurological sequelae.

\section{Discussion-Conclusion}

Activation of the coagulation system, producing hypercoagulability, is one of the most known consequences of SARS-CoV-2 infection, besides pulmonary manifestations [3]. This procoagulant state is due to several mechanisms that include the activation of both extrinsic and intrinsic pathways resulting in an increased factor VIII, fibrinogen, PAI-1, Von Willebran factor, tissue factor

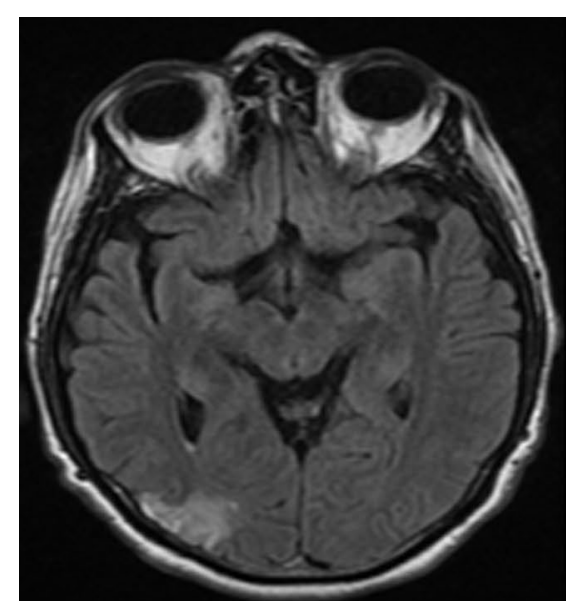

A

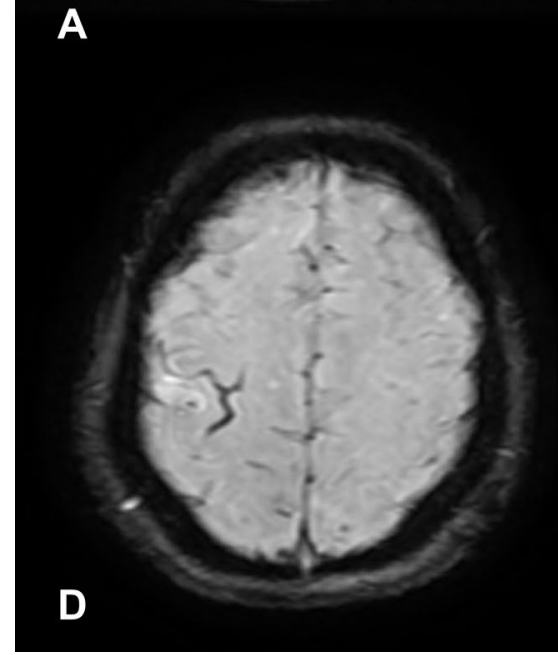

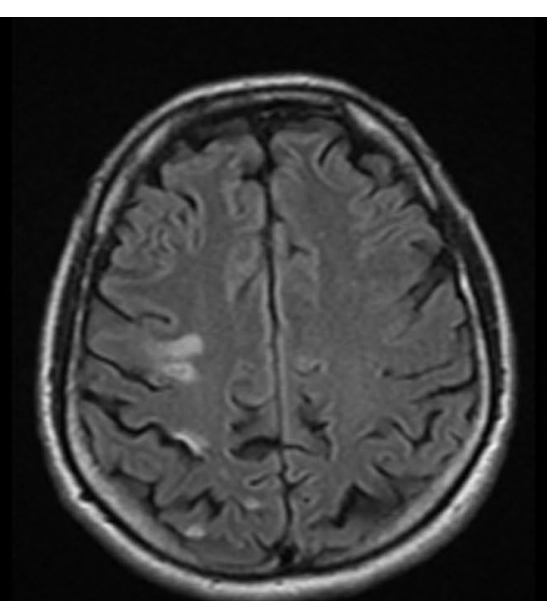

B

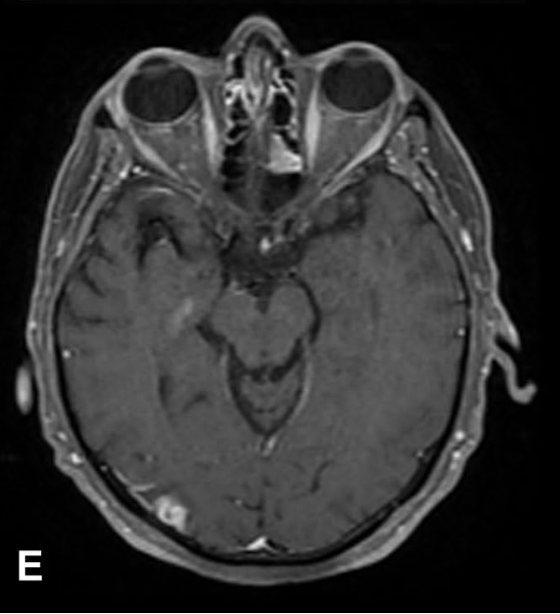

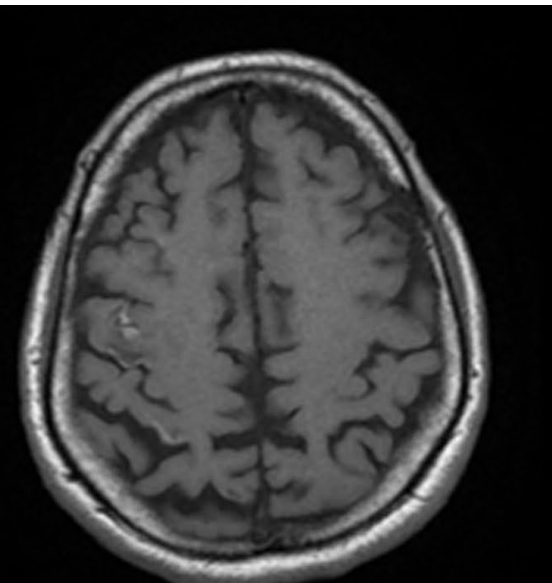

C

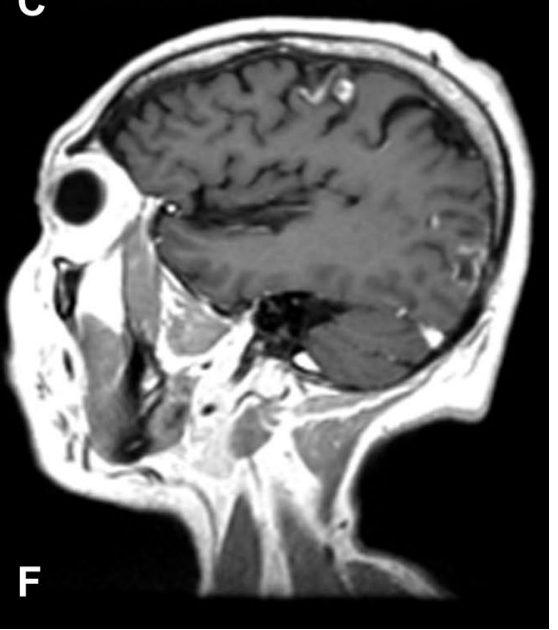

Fig. 1 A MRI and FLAIR T2W images. Cortical and subcortical high T2 signal in the posterior right temporal lobe corresponding to arterial infarct B High T2 signal subcortical in the frontal lobe corresponding to venous infarct $\mathbf{C} \mathrm{T} 1 \mathrm{~W}$ axial view. High subcortical signal due to hemorrhage in venous infarct. D SWI linear low signal corre- sponding to thrombosed cortical frontal vein. E Axial T1W with gadolinium. Cortical and meningeal enhancement typical of an arterial stroke. F Sagittal T1W with gadolinium. Intrinsic T1 enhanncement of the thrombosed frontal vein and in cortex of the posterior cortical temporal stroke 
Fig. 2 A MR with gadolinium. Right transverse and sigmoid sinuses with partial enhancement due to subacute thrombosis. B MR angiography with significant decrease of the venous flow in the right transverse and sigmoid sinuses due to partially recanalized thrombosis

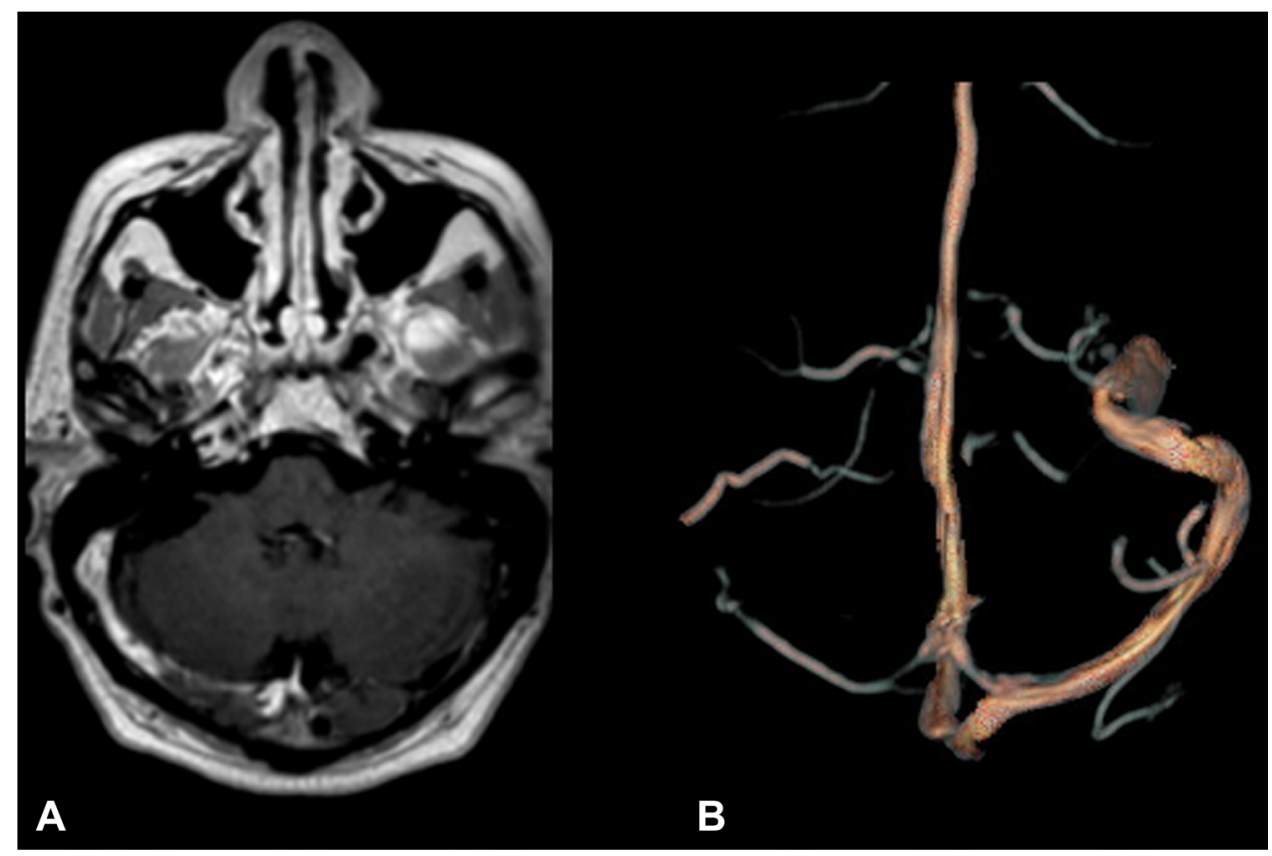

expression, thrombin generation and platelet activation, and a decreased in antithrombin, protein $\mathrm{C}$, and thrombomodulin [4].

All the above converge, triggering episodes like brain thrombosis. In the literature, both arterial and venous strokes have been described independently, presenting either as large artery occlusions, especially in the territory of middle cerebral artery (MCA) [5], or as cerebral venous sinus obstruction, even in the context of an anticoagulated patient [6]. Only one previous case described simultaneous involvement of M1 and M2 segments, and the right sigmoid sinus [2]. This case report is consistent with our patient's findings, in whom the same vascular territories were affected along with thrombosis of the transverse sinus and right jugular vein. However, in the study published by Kananeh et al. [6], other vessels were altered in 4 patients with either arterial or venous clot. These were right internal carotid artery, vein of Galen, straight sinus, and right basal vein of Rosenthal. MCA occlusion was also present in 2 cases [6], making it possibly one of the most frequently affected structure as was seen in the cohort performed by Ntaios et al. [5], where 93 out of 163 patients reported compromise of this vessel, and $22.7 \%$ of the patients with complete diagnostic workup $(n=130)$ had a large-artery stroke. The reason to consider this an arterial stroke is the MRI appearance: cortical and subcortical affection and enhancement of the cortex and in the adjacent meninge which is typical of an arterial infarct. The venous infarct is related with a cortical thrombosed vein adjacent to the frontal lobe and tributary of superior sagittal sinus (Fig. 1D). This frontal infarct is venous due to the MR appearance: subcortical affection with hemorrhage, as is shown in Fig. 1C T1-weighted image.
Evidence suggests that ischemic strokes due to COVID19 have worse functional outcome and higher mortality [5]; therefore, a careful and meticulous diagnosis must be made in order to improve the morbidity and mortality of these patients. Fortunately, for our patient, his outcome was favorable.

Our case report is a clear example of complicity among clinical and radiological findings. Neurological examination orientated towards a stroke and its etiology, and images confirmed the diagnosis and revealed the severity and the extent of the disease.

However, there is not enough data about both arterial and venous brain thrombosis that elucidates the differences in characteristics, outcomes, severity of the disease, response to the treatment, among other variables, between arterial stroke alone and venous and arterial compromise. As was described before, there have already been reports of large cohorts of arterial strokes secondary to COVID19; however, only two cases (including this one) exist of arterial and venous stroke, which prevent us from knowing in depth what factors are related to a concomitant or more extensive involvement.

Throughout time, more is known about COVID-19 manifestations, and more detailed information is under investigation. In this context, our case report could be the starting point of new hypothesis about the matter.

Author Contribution Dr. Bermúdez, Dr. Forero, Dr. Salej, Dr. Gonzalez, and Dr. Toro, contributed to the design and implementation of the research, to the analysis of the results, and to the writing of the manuscript. 
Data Availability The data that support the findings of this study are available from the corresponding author upon reasonable request.

\section{Declarations}

Ethics Approval All procedures performed in studies involving human participants were in accordance with the ethical standards of the institutional and/or national research committee and with the 1964 Helsinki declaration and its later amendments or comparable ethical standards.

Consent to Participate/for Publication Consent to participate and consent for publication were obtained from all individual participants included in the study.

Conflict of Interest The authors declare no competing interests.

\section{References}

1. Singhania N, Bansal S, Nimmatoori DP, Ejaz AA, et al. Current overview on hypercoagulability in COVID-19. Am J Cardiovasc Drugs [Internet]. 123AD;20:393-403. https://doi.org/10.1007/ s40256-020-00431-z
2. Malentacchi M, Gned D, Angelino V, Demichelis S, Perboni A, Veltri A, et al. Concomitant brain arterial and venous thrombosis in a COVID-19 patient. Eur J Neurol. 2020;27(9):e38-9.

3. Bautista-Vargas M, Bonilla-Abadía F, Cañas CA. Potential role for tissue factor in the pathogenesis of hypercoagulability associated with in COVID-19. J Thromb Thrombolysis. 2020;50:479-83. https://doi.org/10.1007/s11239-020-02172-x.

4. Mazzeffi MA, Chow JH, Tanaka K. COVID-19 Associated hypercoagulability: manifestations, mechanisms, and management. Shock. 2021;55(4):465-71.

5. Ntaios G, Michel P, Georgiopoulos G, Guo Y, Li W, Xiong J, et al. Characteristics and outcomes in patients with COVID-19 and acute ischemic stroke: The Global COVID-19 Stroke Registry. Stroke. 2020;(September):254-8.

6. Kananeh MF, Thomas T, Sharma K, Herpich F, Urtecho J, Athar MK, et al. Arterial and venous strokes in the setting of COVID-19. J Clin Neurosci. 2020;79:60-6.

Publisher's Note Springer Nature remains neutral with regard to jurisdictional claims in published maps and institutional affiliations. 